\title{
Design of Extended Channel Ge-source TFET for Low Power Applications
}

\author{
Fatma Shokry $^{1}$, Ahmed Shaker ${ }^{2}$, MK Elsaid ${ }^{1}$, Mohamed Abouelatta ${ }^{{ }^{*}}$ \\ ${ }^{1}$ Electronics and Communication Department, \\ Faculty of Engineering, Ain Shams University, 11517, Cairo, EGYPT \\ ${ }^{2}$ Engineering Physics and Mathematics Department, \\ Faculty of Engineering, Ain Shams University, 11517, Cairo, EGYPT \\ *Corresponding Author
}

DOI : https://doi.org/10.30880/ijie.2020.12.08.018

Received 19 October 2019; Accepted 8 July 2020; Available online 31 August 2020

\begin{abstract}
In this paper, a novel design of a TFET structure using Ge-source and extending a part of the channel into the source is proposed. The DC performance is analyzed by evaluating the ON current, $I_{O N} / I_{O F F}$ ratio and subthreshold swing $(S S)$. Moreover, the high-frequency performance is inspected in terms of transconductance $\left(g_{m}\right)$ and unit-gain cutoff frequency $\left(f_{T}\right)$. All simulations are performed utilizing 2D SILVACO TCAD. It is demonstrated that the ON current and the cut-off frequency can be simultaneously improved by appropriate design of the proposed structure.
\end{abstract}

Keywords: TFET, ambipolar current, Band-To-Band Tunneling (BTBT), transconductance, Subthreshold Swing (SS), cut-off frequency

\section{Introduction}

The continuous scaling down of the conventional MOSFET leads to a superior behavior regarding higher speed, improved high frequency (HF) performance and lower operational power $[1,2]$. However, the miniaturization is obstructed by several unwanted effects that arose in the MOSFET as the device size goes on shrinking [3]. CMOS technology shows certain conditions as the device is scaled more and more in the nanometer regime, out of which power dissipation is an important issue. To overcome the issues regarding power dissipation, specific low power design techniques using CMOS are implemented [4]. Reduction of power dissipation could be achieved by reducing the supply voltage, but this has a disadvantage of increasing the delay.

New devices are being proposed in the literature to overcome the disadvantages resulted from miniaturization. One of the most promising devices in this regard is the tunneling FET (TFET). A TFET is a gated PiN diode that is turned on by applying a gate bias [5]. The gate voltage is used to modulate the width of the tunneling barrier, as the width is controlled by the electric field in the tunneling junction.

The current in conventional MOSFETs is based on the thermionic emission mechanism of charge carriers over a potential barrier. Consequently, keeping the power consumption within an acceptable boundary is one of the most severe difficulties for the conventional MOSFETs at advanced technology nodes. But TFETs avoid this issue by using the band-to-band tunneling (BTBT) mechanism rather than thermal injection to inject carriers into the channel of the device. The result of these mechanisms shows that the minimum subthreshold swing $(S S)$ of the MOSFET is about 60 
$\mathrm{mV} /$ decade at room temperature. In comparison, the $S S$ of TFETs is generally less than $60 \mathrm{mV} / \mathrm{decade}$ at room temperature [6].

Besides exhibiting SS lower than $60 \mathrm{mV} /$ decade, TFETs also have the following advantages: reduced leakage current by using the BTBT mechanism and the similarity in the fabrication process compared with MOSFET [7]. However, TFETs have two critical drawbacks: low ON-current level and ambipolar behavior [8].

The coupling of the gate voltage with the channel potential has a profound impact on the $I_{O N}$ of the TFET. This has motivated researchers to design the transistor gate to improve $I_{O N}$ using some techniques like employing multiple gate structures $[9,10]$ or using a semiconductor heterojunction of different materials to have lower effective bandgap and hence higher $I_{O N}$ and lower subthreshold swing [11]. In this respect, low bandgap semiconductors, such as InAs [12] and $\mathrm{Mg}_{2} \mathrm{Si}$ [13], have been utilized. Such materials demonstrated higher tunneling rates than those in silicon because of their small bandgap, leading to a higher ON/OFF current ratio [14, 15].

One of the most attractive alternative materials used in TFETs is Germanium $(\mathrm{Ge})$. Ge has attracted the attention of device engineers due to having a low bandgap $\left(E_{g}=0.67 \mathrm{eV}\right)$ and high carrier mobility. These characteristics result in a higher $I_{O N}[16,17]$. Some research works have considered the transfer characteristics and capacitance of Ge TFET but did not investigate the transconductance and the cutoff frequency of the device that are considered the main analog/RF parameters $[18,19]$.

In this paper, a new design of a TFET structure is proposed in which a part of the channel is extended into the source. The Ge material is used to serve as a source, while silicon is employed as the channel and drain material. By using the proposed structure, the ON current of Ge-source TFET is improved as well as the transconductance. The enhancement in transconductance boosts the cutoff frequency, as will be shown herein.

The paper is structured as follows. Following the introduction, section 2 presents the simulation methodology and device structure. The simulation results are discussed in section 3. Section 4 is devoted to the parametric analysis. The final section is devoted to the paper conclusion.

\section{Simulation Methodology and Device Structure}

All simulations, presented in this work, have been performed using SILVACO 2D ATLAS device simulator. The models used in the simulations are listed in Table 1. Firstly, the BTBT model is calibrated against the work in [20], Fig. 1 shows the calibration results. The electron and hole tunneling masses have been refined as $m_{e}=0.11$ and $m_{h}=0.17$, respectively. These values are adjusted to achieve best-fit between simulation and fully quantum simulation results in $[20]$.

Table 1 - Physical models used in the simulation

\begin{tabular}{ll}
\hline Physical Model & Description \\
\hline Mobility & CVT: Enables transverse field, doping and temperature-dependent parts of mobility \\
Statistics & FERMI: To consider the highly doped regions, Fermi-Dirac statistics are adopted \\
Tunneling & BBT.NONLOCAL: The nonlocal BTBT model is used to consider the lateral tunneling through the \\
& channel. \\
Drift-Diffusion & Used for the transfer of holes and electrons \\
\hline
\end{tabular}

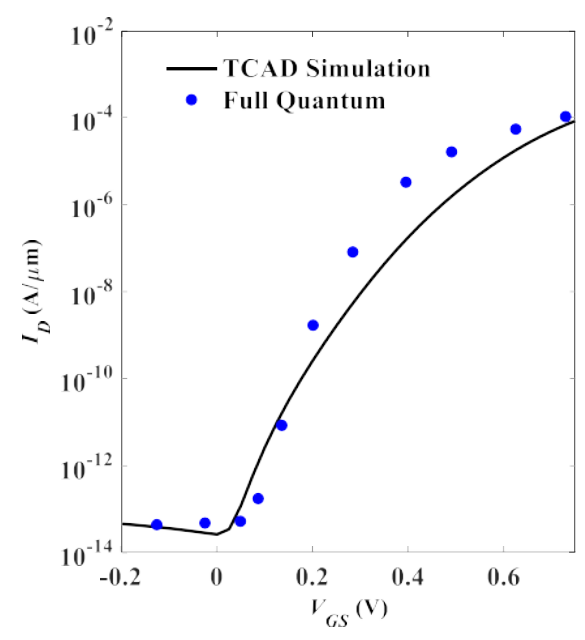

Fig. 1 - TCAD model simulation calibrated vs the full quantum simulation in REF [20] 
A cross-sectional schematic of an n-type double gate (DG) TFET is shown in Fig. 2. The structure comprises a Ge $\mathrm{p}^{+}$source, $\mathrm{Si}$ intrinsic channel, and $\mathrm{Si}^{+}$drain. All the junctions are assumed to be abrupt with uniform doping profiles. Further, in the proposed structure, the channel is extended into the source region with a length defined by $L_{o v}$. Fig. 2(a) presents the schematic diagram showing different regions and the design parameter $L_{o v} . L_{o v}$ affects the band bending at the interface between the source and channel regions and hence the tunneling rates. As a result, $L_{o v}$ has a direct influence on the electrical behavior of the transistor. Its value can be varied from 0 up to the source length. In the following simulation, $L_{o v}$ is varied from 0 (conventional case) to $16 \mathrm{~nm}$.

The biasing conditions are $V_{D S}=0.5 \mathrm{~V}$ and $-0.2<V_{G S}<0.5 \mathrm{~V}$ at $T=300 \mathrm{~K}$. The geometrical parameters and doping levels are listed in Table 2. A high doping concentration of $1 \times 10^{20} \mathrm{~cm}^{-3}$ is adopted for the source region to enhance the tunneling current [21]. Meanwhile, the channel doping is low enough $\left(1 \times 10^{16} \mathrm{~cm}^{-3}\right)$ to produce a p-i-n configuration. Further, the drain doping is not so high to suppress the ambipolar current [22]. Its value is $1 \times 10^{18} \mathrm{~cm}^{-3}$, which is relatively high, to ensure a drain electrode Ohmic contact [22]. The work function of the gate is taken to be 4.4 $\mathrm{eV}$ to ensure that the current starts to increase after $V_{G S}=0 \mathrm{~V}$ [23]. Moreover, the gate oxide material used is $\mathrm{SiO}_{2}$ (with dielectric constant $=3.9$ ) whose thickness is $t_{o x}=1 \mathrm{~nm}$.

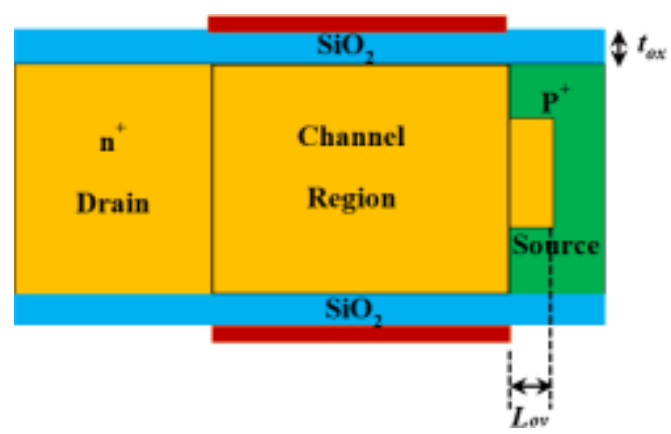

$a$

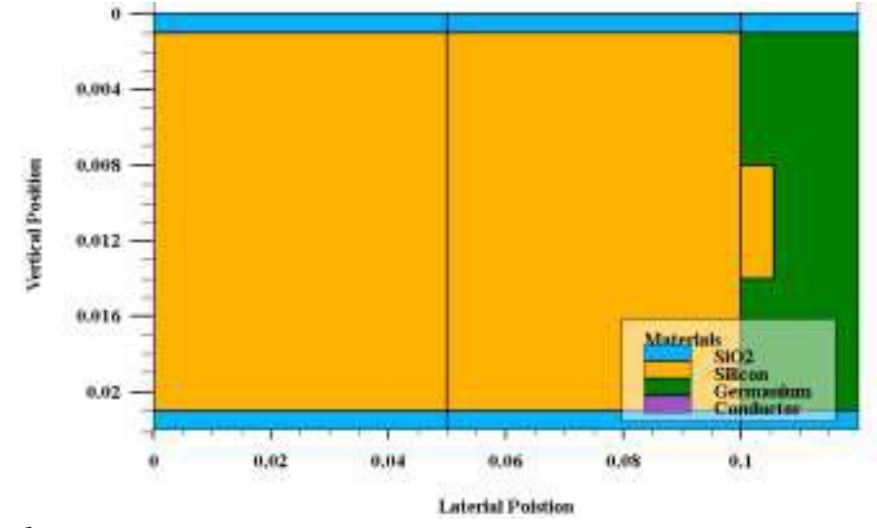

$b$

Fig. 2 - Proposed TFET device Structure (All dimensions are given in $\mu \mathrm{m}$ ) (a) Schematic diagram showing different regions and the design parameter $L_{o v}$ and (b) Output structure from SILVACO device simulator

Table 2 - Main design parameters in device simulation

\begin{tabular}{ll}
\hline Parameter & Value \\
\hline Source Length $\left(L_{s}\right)$ & $20 \mathrm{~nm}$ \\
Channel Length $\left(L_{c h}\right)$ & $50 \mathrm{~nm}$ \\
Drain Length $\left(L_{d}\right)$ & $50 \mathrm{~nm}$ \\
Gate oxide thickness $\left(t_{o x}\right)$ & $1 \mathrm{~nm}$ \\
Source doping (p-type) $\left(N_{s}\right)$ & $1 \times 10^{20} \mathrm{~cm}^{-3}$ \\
Drain doping (n-type) $\left(N_{d}\right)$ & $1 \times 10^{18} \mathrm{~cm}^{-3}$ \\
Channel doping (p-type) $\left(N_{c h}\right)$ & $1 \times 10^{16} \mathrm{~cm}^{-3}$ \\
Gate work function $\left(\Phi_{g}\right)$ & $4.4 \mathrm{eV}$ \\
\hline
\end{tabular}

\section{Simulation Results}

In the following simulations, the overlap distance $\left(L_{o v}\right)$ of the channel into the source is varied to inspect its influence on the device electrical behavior. Firstly, the nonlocal BTB tunneling rates are shown in Fig. 3 for two values of $L_{o v}(6 \mathrm{~nm}$ and $12 \mathrm{~nm})$ compared to the conventional structure with no channel extension. It can be inferred that the proposed structure provides much higher rates than the conventional one (either for holes or electrons). These high tunneling rates indicate higher $\mathrm{ON}$ current when using the proposed structure.

Now, a comparison between the transfer characteristics of the conventional and proposed TFET for two values of $L_{o v}$ is shown in Fig. 4. It is observed that by increasing the overlap length, the ON current becomes higher. The ON current is doubled at $V_{G S}=0.5 \mathrm{~V}$ and $L_{o v}=12 \mathrm{~nm}$. At the same time, the OFF current substantially remains constant, so the ON/OFF current ratio increases by the same factor when the overlapping increases. The results indicate that, at $L_{o v}$ $=12 \mathrm{~nm}$, the ON current is about 2.5 of the conventional ON current at the ON state for which $V_{G S}=0.5 \mathrm{~V}$. 


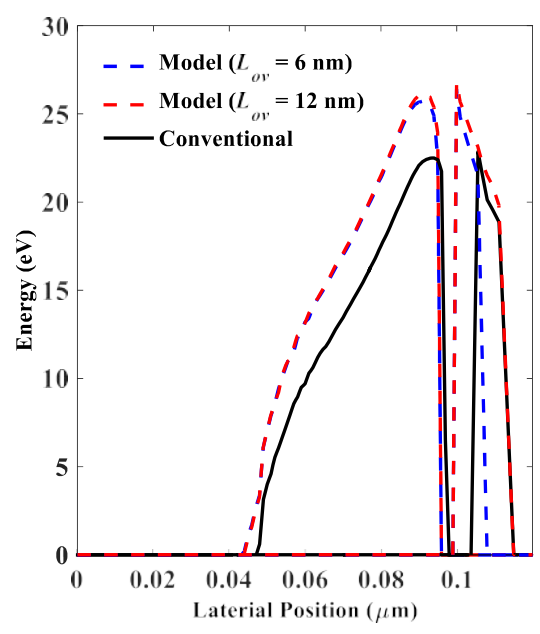

Fig. 3 - BTB electron and hole tunneling rates for the proposed structure with different values of $L_{o v}$ (at $V_{D S}$ $=0.5$ and $\left.V_{G S}=0.5 \mathrm{~V}\right)$
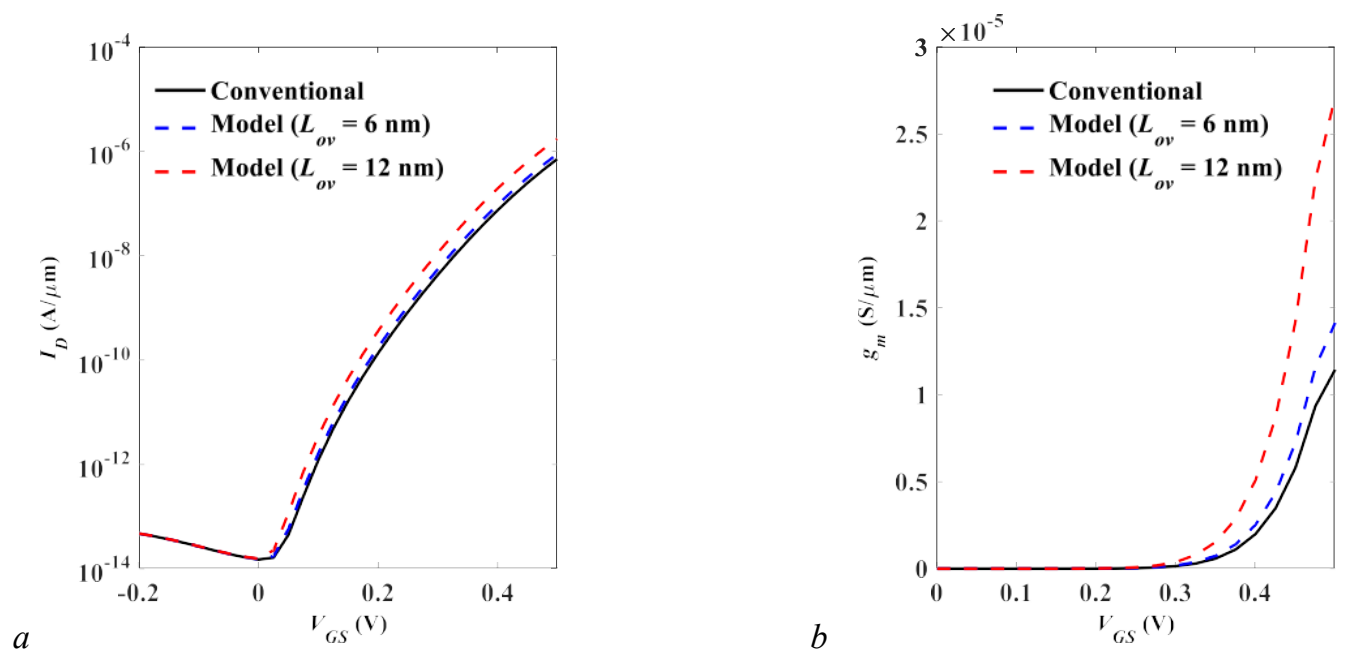

Fig. 4 - Transfer characteristics of the conventional and proposed TFET structures with different overlap lengths at $V_{D S}=0.5 \mathrm{~V}$ (a) $\log$ scale and (b) linear scale

Next, the high-frequency parameters are inspected. The high-frequency performance is studied considering the transconductance $\left(g_{m}\right)$ and gate capacitance $\left(C_{g g}\right)$. Then, the unit gain cutoff frequency $\left(f_{T}\right)$ is calculated based on the previous two parameters. Fig. 5(a) illustrates the variation of the intrinsic total gate capacitance $\left(C_{g g}\right)$ of the proposed structure at $V_{D S}=0.5 \mathrm{~V}$. The result is compared with the gate capacitance of the conventional TFET structure. It is evident from the figure that $C_{g g}$ of the proposed structure is lowered with the increase of overlap length. When $L_{o v}=12$ $\mathrm{nm}, C_{g g}$ is lowered by about $7 \%$ from its value for the conventional structure with no overlap (Given $V_{G S}=0.5 \mathrm{~V}$ ).

The transconductance is given by the rate of increase of the drain current with the gate-to-source voltage [24]. It can be formulated as,

$$
g_{m}=\frac{\partial I_{D}}{\partial V_{G S}}
$$

The variation in transconductance is shown in Fig. 5(b). The proposed structure shows higher transconductance as compared to conventional TFET because of an increase in tunneling volume in the channel. Now, the cutoff frequency is approximated by the ratio of the transconductance to the total gate capacitance [25]. It can be given as, 


$$
f_{T}=\frac{g_{m}}{2 \pi C_{g s} \sqrt{1+2 C_{g d} / C_{g s}}} \approx \frac{g_{m}}{2 \pi\left(C_{g d}+C_{g s}\right)}=\frac{g_{m}}{2 \pi C_{g g}}
$$

Where $g_{m}$ is the transconductance and $C_{g g}$ is the overall gate capacitance. From the previous equation, $f_{T}$ is improved by increasing the transconductance and reducing the total gate capacitance [26, 27].
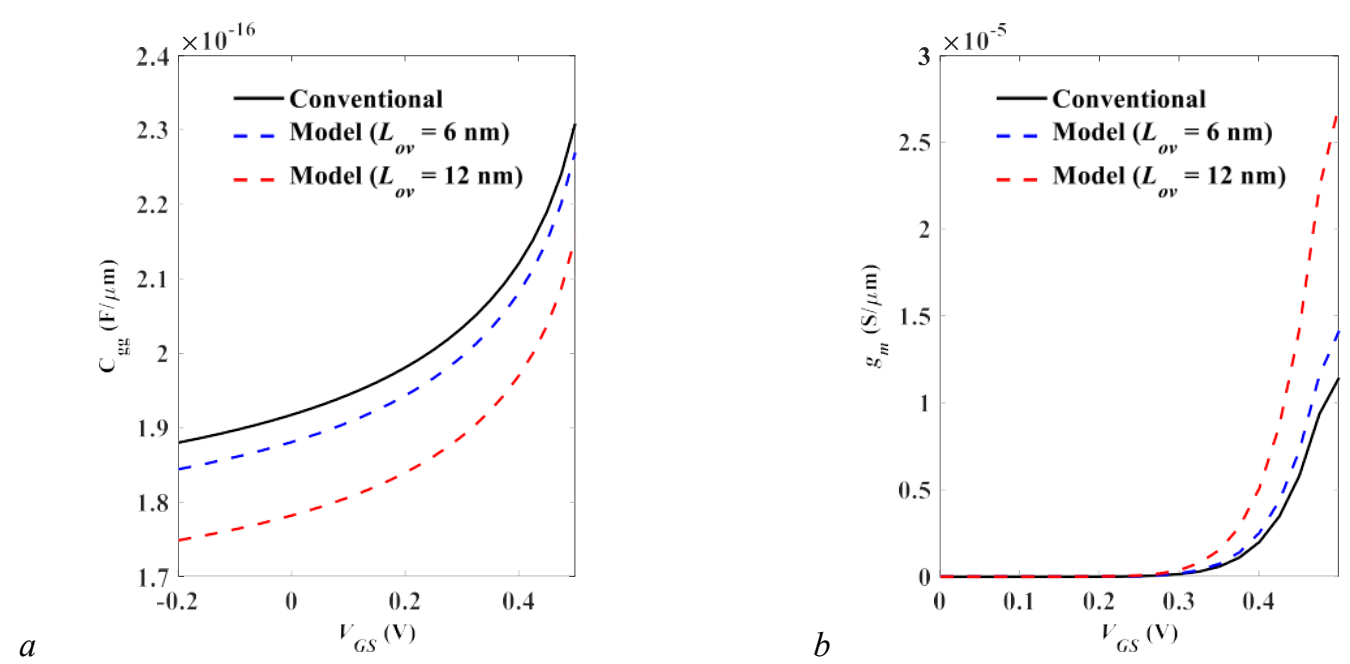

Fig. 5 - (a) Variation of the intrinsic total gate capacitance and (b) transconductance as a function of gate voltage for the proposed structure (at $V_{D S}=0.5 \mathrm{~V}$ )

Fig. 6 shows the variation of the cutoff frequency of the proposed structure as a function of gate voltage at $V_{D S}=0.5 \mathrm{~V}$. The cutoff frequency of the proposed structure increases with the increase of $L_{o v} . f_{T}$ is increased by a factor of 2.7 at $V_{G S}$ $=0.5 \mathrm{~V}$ and $L_{o v}=12 \mathrm{~nm}$. This behavior is due to increasing the ratio of $g_{m}$ and $C_{g g}$ (as can be depicted from Fig. 5).

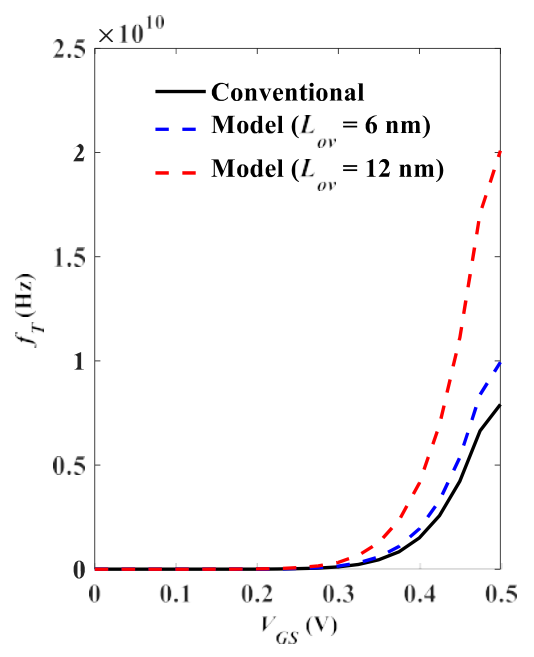

Fig. 6 - Cutoff frequency variation vs gate voltage at $V_{D S}=0.5 \mathrm{~V}$

\section{Parametric Analysis}

In order to design the proposed structure, we examine the variation of $L_{o v}$ from $6 \mathrm{~nm}$ to $16 \mathrm{~nm}$ to investigate the influence of the channel extension into the source. The main parameters studied to measure this impact are $S S, I_{O N}$ and $f_{\text {Tmax }}$. The $S S$ can be defined as the gate voltage required to change the drain current by one decade. It can be determined as the inverse of the slope of the $\left(\log I_{D}\right)$ vs $V_{G S}$ curve in the subthreshold exponential region [28]. It can be formulated as, 


$$
S S=\frac{\partial V_{G S}}{\partial\left(\log I_{D}\right)}
$$

Where the slope is determined by the partial differentiation of $\left(\log I_{D}\right)$ w.r.t. $V_{G S}$. Fig. 7(a) shows the $S S$ variation with $L_{o v}$. As can be seen, $S S$ decreases when increasing $L_{o v}$. So, there is an improvement in $S S$ as a smaller $S S$ leads to more improved dynamic performance. Moreover, Fig. 7(b) and Fig. 7(c) show an improvement in the ON current and the maximum cutoff frequency, $f_{\text {Tmax }}$, with increasing the overlapping length. By increasing the overlapping length from $6 \mathrm{~nm}$ to $16 \mathrm{~nm}, S S$ is decreased from $33 \mathrm{mV} /$ decade to less than $30 \mathrm{mV} /$ decade, $I_{O N}$ is increased from $1 \mu \mathrm{A} / \mu \mathrm{m}$ to 5 $\mu \mathrm{A} / \mu \mathrm{m}$ and $f_{\text {Tmax }}$ is increased from $10 \mathrm{GHz}$ to $65 \mathrm{GHz}$.
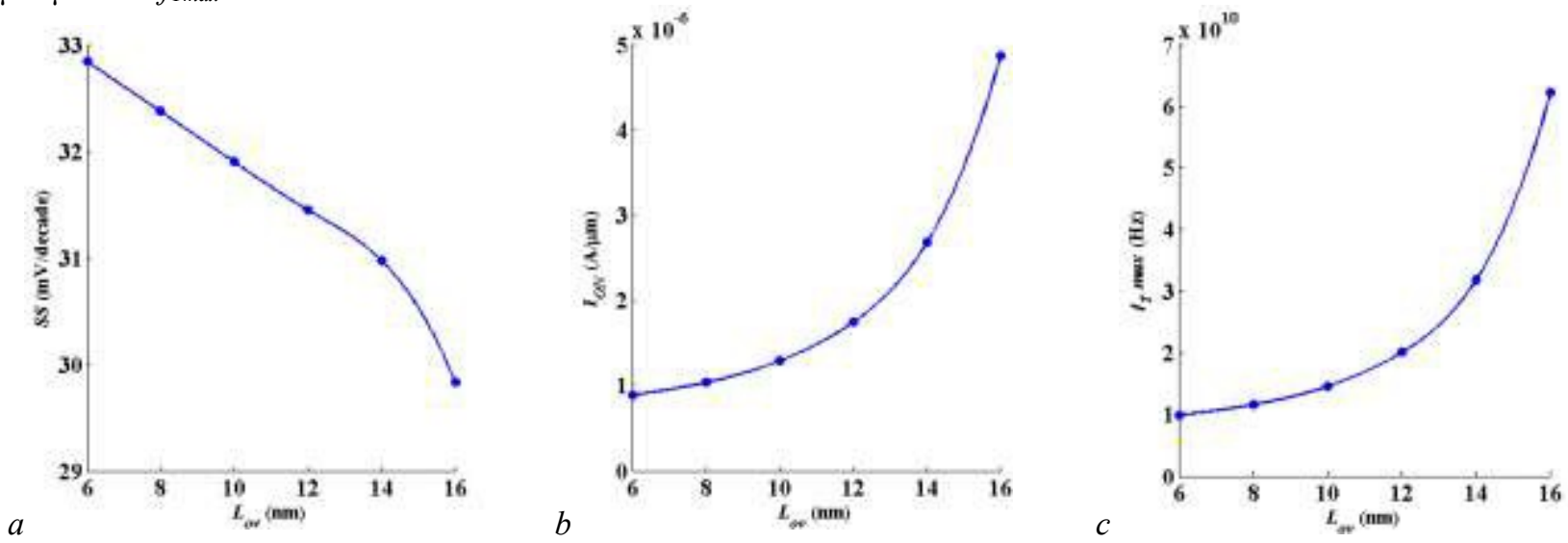

Fig. 7 - Variation of performance parameters with $L_{o v}\left(\right.$ at $V_{D S}=0.5 \mathrm{~V}$ and $V_{G S}=0.5 \mathrm{~V}$ ): (a) sub-threshold swing, (b) ON current and (c) maximum cut-off frequency

\section{Conclusion}

In this paper, by using 2D TCAD simulations, the impact of extending the channel into the Ge-source region of TFET has been studied to control the ON current while improving the high-frequency performance. It is demonstrated that by overlapping a part of the channel into the source region in a TFET, the ON current increases. In addition, the capacitance, transconductance and, in turn, the cutoff frequency are improved. By increasing the overlapping length, $L_{o v}$, from $6 \mathrm{~nm}$ to $16 \mathrm{~nm}, S S$ is decreased from $33 \mathrm{mV} /$ decade to less than $30 \mathrm{mV} /$ decade, $I_{O N}$ is increased by a factor of 5 and $f_{\text {Tmax }}$ is increased by a factor of 6.5. Based on this TCAD simulation study, the proposed structure could be used effectively for low power applications.

\section{Acknowledgement}

The authors would like to express their gratitude towards Prof. Dr. A. Zekry of Microelectronic Research Centre for his valuable discussion throughout this work.

\section{References}

[1] Kilchytska, V., Neve, A., Vancaillie, L., Levacq, D., Adriaensen, S., van Meer, H., \& Flandre, D. (2003). Influence of device engineering on the analog and RF performances of SOI MOSFETs. IEEE Transactions on Electron Devices, 50, 577-588.

[2] Abou-Allam, E., Manku, T., Ting, M., \& Obrecht, M. S. (2000, May). Impact of technology scaling on CMOS RF devices and circuits. In Proceedings of the IEEE 2000 Custom Integrated Circuits Conference (Cat. No. 00CH37044) (pp. 361-364). IEEE.

[3] Swahn, B., \& Hassoun, S. (2006, July). Gate sizing: FinFETs vs 32nm bulk MOSFETs. In Proceedings of the 43rd annual Design Automation Conference (pp. 528-531).

[4] Rabaey, J. M., Chandrakasan, A. P., \& Nikolić, B. (2003). Digital integrated circuits: a design perspective (Vol. 7). Upper Saddle River, NJ: Pearson Education.

[5] Khatami, Y., \& Banerjee, K. (2009). Steep subthreshold slope n-and p-type tunnel-FET devices for low-power and energy-efficient digital circuits. IEEE Transactions on Electron Devices, 56, 2752-2761. 
[6] Akarvardar, K., Elata, D., Parsa, R., Wan, G. C., Yoo, K., Provine, J., \& Wong, H. S. (2007, December). Design considerations for complementary nanoelectromechanical logic gates. In 2007 IEEE International Electron Devices Meeting (pp. 299-302). IEEE.

[7] Aswathy, M., Biju, N. M., \& Komaragiri, R. (2013, August). Comparison of a 30nm tunnel field effect transistor and CMOS inverter characteristics. In 2013 Third International Conference on Advances in Computing and Communications (pp. 149-152). IEEE.

[8] Salem, N., Ossaimee, M., Shaker, A., \& Abouelatta, M. (2018). Electrical Characteristics of T-CNTFET: PartiallyGated Channel vs Doping Engineering. Ecs Journal of Solid State Science and Technology, 7, M23.

[9] Morita, Y., Mori, T., Migita, S., Mizubayashi, W., Tanabe, A., Fukuda, K. \& Masahara, M. (2014). Performance enhancement of tunnel field-effect transistors by synthetic electric field effect. IEEE Electron Device Letters, 35 , 792-794.

[10] Toh, E. H., Wang, G. H., Samudra, G., \& Yeo, Y. C. (2007). Device physics and design of double-gate tunneling field-effect transistor by silicon film thickness optimization. Applied Physics Letters, 90, 263507.

[11] Wang, P. Y., \& Tsui, B. Y. (2015). Band engineering to improve average subthreshold swing by suppressing low electric field band-to-band tunneling with epitaxial tunnel layer tunnel FET structure. IEEE Transactions on Nanotechnology, 15, 74-79.

[12] Moselund, K. E., Cutaia, D., Schmid, H., Borg, M., Sant, S., Schenk, A., \& Riel, H. (2016). Lateral InAs/Si p-type tunnel FETs integrated on $\mathrm{Si}$ - part 1: experimental devices. IEEE Transactions on Electron Devices, 63, 42334239.

[13]Elnaggar, M., Shaker, A., \& Fedawy, M. (2019). A comprehensive investigation of TFETs with semiconducting silicide source: impact of gate drain underlap and interface traps. Semiconductor Science and Technology, 34, 045015.

[14] Mehta, J. U., Borders, W. A., Liu, H., Pandey, R., Datta, S., \& Lunardi, L. (2015). III-V tunnel FET model with closed-form analytical solution. IEEE Transactions on Electron Devices, 63, 2163-2168.

[15] Wang, L., Yu, E., Taur, Y., \& Asbeck, P. (2010). Design of tunneling field-effect transistors based on staggered heterojunctions for ultralow-power applications. IEEE Electron Device Letters, 31, 431-433.

[16] Agopian, P. G., Martino, M. D., dos Santos, S. D., Neves, F. S., Martino, J. A., Rooyackers, R., \& Claeys, C. (2014). Influence of the source composition on the analog performance parameters of vertical nanowireTFETs. IEEE Transactions on Electron Devices, 62, 16-22.

[17] Verhulst, A. S., Vandenberghe, W. G., Maex, K., \& Groeseneken, G. (2007). Tunnel field-effect transistor without gate-drain overlap. Applied Physics Letters, 91, 053102.

[18] Han, T., Liu, H., Wang, S., Chen, S., Li, W., Yang, X., Yang, K. (2019). Design and Investigation of the JunctionLess TFET with $\mathrm{Ge} / \mathrm{Si}_{0.3} \mathrm{Ge}_{0.7} / \mathrm{Si}$ Heterojunction and Heterogeneous Gate Dielectric. Electronics, 8, p. 476.

[19] Kim, S. H., Agarwal, S., Jacobson, Z. A., Matheu, P., Hu, C., \& Liu, T. J. K. (2010). Tunnel field effect transistor with raised germanium source. IEEE Electron Device Letters, 31, 1107-1109.

[20] Jain, P., Rastogi, P., Yadav, C., Agarwal, A., \& Chauhan, Y. S. (2017). Band-to-band tunneling in $\Gamma$ valley for Ge source lateral tunnel field effect transistor: thickness scaling. Journal of Applied Physics, 122, 014502.

[21] Boucart, K., \& Ionescu, A. M. (2007). Double-gate tunnel FET with high-k gate dielectric. IEEE transactions on electron devices, 54, 1725-1733.

[22] Shaker, A., El Sabbagh, M., \& El-Banna, M. M. (2017). Influence of drain doping engineering on the ambipolar conduction and high-frequency performance of TFETs. IEEE Transactions on Electron Devices, 64, 3541-3547.

[23] Elgamal, M., Sinjab, A., Fedawy, M., \& Shaker, A. (2019). Effect of doping profile and the work function variation on performance of double-gate TFET. International Journal of Integrated Engineering, 11, 40-46.

[24] Ranjan, R., Pradhan, K. P., \& Sahu, P. K. (2016). A comprehensive investigation of silicon film thickness (T SI) of nanoscale DG TFET for low power applications. Advances in Natural Sciences: Nanoscience and Nanotechnology, 7, 035009.

[25] Wang, W., Wang, P. F., Zhang, C. M., Lin, X., Liu, X. Y., Sun, Q. Q., \& Zhang, D. W. (2013). Design of U-shape channel tunnel FETs with SiGe source regions. IEEE Transactions on Electron Devices, 61, 193-197.

[26] Shaker, A., Ossaimee, M., Zekry, A., \& Abouelatta, M. (2015). Influence of gate overlap engineering on ambipolar and high frequency characteristics of tunnel-CNTFET. Superlattices and Microstructures, 86, 518-530.

[27] Pown, M., \& Lakshmi, B. (2016). Investigation of $\mathrm{f}_{\mathrm{t}}$ and $\mathrm{f}_{\max }$ in $\mathrm{Si}$ and $\mathrm{Si}_{1-\mathrm{x}} \mathrm{Ge}_{\mathrm{x}}$ based single and dual material double-gate Tunnel FETs for RF applications. Advances in Natural Sciences: Nanoscience and Nanotechnology, 7, 025006.

[28] Ashwin, S. R., Sreejith, S., \& Sajeshkumar, U. (2015). TCAD Design of Tunnel FET Structures and Extraction of Electrical Characteristics. International Journal of Science and Research (IJSR), 4, 2447-2451. 